\title{
Una nueva especie de Miconia (Melastomataceae) de hojas rojizas de los andes de Colombia
}

\author{
Humberto Mendoza-Cifuentes ${ }^{1, *}$, Juan Mauricio Posada-Herrera ${ }^{2}$ \\ ${ }^{1}$ Instituto Alexander von Humboldt, Herbario FMB, Claustro de San Agustín, Villa de Leyva, Colombia \\ ${ }^{2}$ Herbario Universidad del Valle CUVC, Cali, Colombia
}

\begin{abstract}
Resumen
Se describe e ilustra Miconia brigittei (Melastomataceae), una nueva especie endémica de los andes de Colombia. Esta especie se asocia con Miconia sección Amblyarrhena y se caracteriza por el color rojizo de las hojas en los ejemplares de herbario, hojas 5 plinervias, domacios pubescentes en la base de las venas secundarias por el envés, pétalos reflexos, conectivo de la antera minutamente prolongado en la base dorsal, y ovario 3-locular. Crece a lo largo de la Cordillera Occidental y centro-norte de la Cordillera Central, entre los 1330 y 2020 m de elevación, en zonas de bosque Subandino y Andino. Por su rango de distribución es categorizada como vulnerable. (C) 2018. Acad. Colomb. Cienc. Ex. Fis. Nat.
\end{abstract}

Palabras clave: Sección Amblyarrhena; Bosques Andinos; Bosque Subandino; Flora de Colombia.

A new species of Miconia (Melastomataceae) of reddish leaves from the Andes of Colombia

\begin{abstract}
Miconia brigittei (Melastomataceae), a new species endemic to the Andes of Colombia, is described and illustrated. This species is placed in Miconia section Amblyatrrhena and is characterized by the reddish color of the leaves in dried specimens, 5-plinervate leaves, tuff domatia at the base of the secondary veins in the abaxial leaf surface, reflexed petals, connective of the anther minutely prolonged in the dorsal base, and 3-locular ovary. It grows along the Western Cordillera and north-central Cordillera Central, between 1330 and 2020 m elevation, in areas of Sub Andean and Andean Forest. Considering the distribution range, it is categorized as vulnerable. (C) 2018. Acad. Colomb. Cienc. Ex. Fis. Nat.
\end{abstract}

Key words: Amblyarrhena section; Andean forest; Flora of Colombia; Subandean forest.

\section{Introducción}

Miconia Ruiz \& Pav. (1794: 60), con cerca de 1100 especies, es el género de plantas leñosas de las angiospermas más diverso con distribución restricta al neotrópico (Goldenberg, et al., 2008, 2013; Michelangeli, 2016). Colombia es el país con mayor número de especies del género, ya que alberga el $36 \%$ de sus taxones, con alrededor de 380 especies (Almeda, et al., 2016). En especial la región andina colombiana es ampliamente diversa en este género con alrededor de 270 especies (Almeda, et al., 2016).

Los herbarios de Colombia, la mayoría de ellos asociados a la región andina, cuentan con abundantes colecciones del género Miconia indeterminadas, algunas de las cuales representan nuevas especies o registros para el país, por lo que el número nacional de especies de Melastomataceae constantemente se incrementa.

La presente novedad taxonómica es producto de la revisión de colecciones de herbarios regionales, como es el herbario FMB, pero también de recolecciones directa de campo. La reciente expedición biológica a la Cordillera Central del departamento de Antioquia, en el marco del proyecto
Colombia BIO financiado por Colciencias y desarrollado por el Instituto Alexander von Humboldt, permitió recolectar ejemplares de este nuevo taxón, lo que ayudó a constatar su novedad. En este artículo se describe y nombra esta especie en reconocimiento a la dirección del Instituto Alexander von Humboldt por apoyar la realización de inventarios biológicos y el trabajo de curatoría básica en algunos de los herbarios nacionales.

\section{Materiales y métodos}

A partir de revisiones de colecciones del herbario FMB y COL, se identificaron ejemplares asociados con este nuevo taxón. Se revisó literatura pertinente al género Miconia en Colombia, Ecuador y Perú, la base de datos del herbario COL, el catálogo de Melastomataceae de Colombia (Almeda, et al., 2016) y la flora de Mesoamericana (Almeda, 2009).

\footnotetext{
*Correspondencia:

Humberto Mendoza-Cifuentes, hmendoza@humboldt.org.co

Recibido: 13 de enero de 2018

Aceptado: 31 de marzo de 2018

Editor: Elizabeth Castañeda
} 
Complementariamente, se consultaron las imágenes tipo de la totalidad de las especies de Miconia de Colombia en la base de datos de Global Plants JSTOR (http://plants.jstor. org/search?plant).

Posteriormente, se procedió a realizar la descripción, ilustración del nuevo taxón y la identificación de las especies más afines dentro del género Miconia. Para la descripción se realizaron medidas de las partes vegetativas y de la inflorescencia en material de herbario y utilizando un calibrador digital de $0,01 \mathrm{~mm}$ de precisión marca Mitutoyo (Series 500). Las medidas de las partes florales se basaron en flores secas rehidratas procedentes de los ejemplares tipo. Se realizaron medidas de las partes florales y tricomas en un microscopio Leica S8AP0. Los tipos de tricomas se identifican con base en el atlas de tricomas de Melastomataceae de Wurdack (1986). Finalmente, se evalúo el estado de conservación del nuevo taxón con base en los criterios de amenaza de la IUCN $(2012,2014)$.

\section{Resultados}

Miconia brigittei Humberto Mend. \& Posada-Herrera, sp. nov. (Figuras 1, 2).

Miconia brigittei is recognized by leaves drying red in herbarium specimens, 5-plinervate, leaves, the presence of tuff domatia at the base of the secondary veins on the abaxial leaf surface, reflexed petals, simple connective minute prolonged at the base, anther with an apical pore, and 3-locular ovary.

TIPO: Colombia. Nariño. Municipio de Ricaurte, Reserva Natural La Planada, parcela permanente de 25 ha.

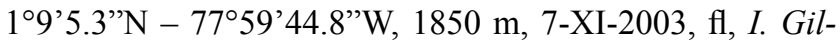
Ch.730 (Holotipo: FMB).

Arbustos o árboles pequeños de 2,5 a 8,0 m de altura. Ramas subcuadrangulares, acanaladas; entrenudos de 3,0$5,6 \mathrm{~cm}$ de largo. Entrenudos y peciolos glabros o con ralos tricomas dendríticos cortos, de $0,3-0,5 \mathrm{~mm}$ de largo, caducos; ejes de la inflorescencia con cubierta densa de tricomas similares a los de ramas, de 0,07-0,12 de largo; envés glabro o con ralos tricomas ramificados cortos y caducos sobre las venas primarias, en las axilas de las venas secundarias en la base de la lámina presenta domacios conformados por tricomas filamentosos ligeramente rugosos, de 1,1-1,5 $\mathrm{mm}$ de largo. Hojas isofilas; peciolos 1,5-4,5 cm de largo, acanalados. Lámina foliar de 11,5-20 × 4,2-10,2 cm, elíptica, obovada-elíptica u ovada; base obtusa a redondeada; ápice acuminado, acumen de $0,6-1,8 \mathrm{~cm}$ de largo; margen entera; haz glabra; envés glabro o con ralos tricomas ramificados cortos. Nervadura con dos pares de venas secundarias acompañando a la vena media, plinervias, el par superior plinervio a $0,2-1,7 \mathrm{~cm}$; con $35-46$ pares de venas terciaria aledañas a la vena media. Inflorescencia paniculada, terminal, de $8-20 \mathrm{~cm}$ de largo, con 80 hasta 150 flores; ejes cuadrangulares; eje central con 4-8 nudos de ramificación; pedúnculo de 2,7-5,0 cm de largo, algunas veces ausente; primer internodo de $2-5 \mathrm{~cm}$ de largo; paracladios basales de 3,5-12,0 cm de largo, con 2-3 niveles de ramificación; ápices trifoloros; brácteas en el primer nudo semejante a hojas pequeñas, con lámina elíptica u obovadoelíptica y de 6,0-13,0 × 2,3-5,0 cm; bracteolas filiformes u oblongas, de $0,8-1,4 \mathrm{~mm}$ de largo, caducas desde botones florales. Flores 5-meras, diplostemonas, pediceladas; pedicelo $0,6-1,0 \mathrm{~mm} \log$. Hipanto campanulado, tálamo de $0,78-0,81 \mathrm{~mm}$ de largo; externamente con cubierta de tricomas ramificados cortos como en la inflorescencia o con tricomas adpresos; tricomas de 0,06 $\mathrm{mm}$ de largo. Cáliz lobulado, externamente con indumento similar al hipantoovario; internamente con tricomas amorfos adpresos; tubo del cáliz, de 0,34-0,36 mm de largo; lóbulos anchamente redondeados, de margen membranosas y fimbriada, de 0,2 $0,3 \times 0,8 \mathrm{~mm}$; diente dorsal diminuto, de $0,2 \mathrm{~mm}$ de largo, con tricomas ramificados cortos. Corola con pétalos reflexos en flores abiertas; pétalos oblongos, de 1,8-2,0 × 0,7-0,9 mm, glabros, blancos, apicalmente con bordes enrollados que dan la apariencia de un ápice agudo. Estambres isomorfos; filamentos aplanados basalmente, 1,4-1,6 $\mathrm{mm}$ de largo y $0,3-0,4 \mathrm{~mm}$ ancho en la base, glabros; anteras amarillas, oblongas, $0,8-1,0 \times 0,37-0,50 \mathrm{~mm}$ y $0,3-0,4 \mathrm{~mm}$ grosor, con un poro apical de $0,15-0,18 \mathrm{~mm}$ de diámetro; conectivo prolongado levemente en la base dorsal, 0,12 $0,14 \times 0,14-0,16$, minutamente bilobado. Ovario 3-locular, completamente fusionado al hipanto, $0,97-1,10 \times 0,95$ $1,00 \mathrm{~mm}$, externamente con cubierta similar al hipanto; ápice redondeado, $0,12-0,15 \mathrm{~mm}$ de largo, con ralos tricomas rugosos alrededor del estilo de $0,06 \mathrm{~mm}$ de largo. Estilo columnar, de 4,1-4,5 $\mathrm{mm}$ de largo y $0,25-0,27 \mathrm{~mm}$ de diámetro en la base, engrosado hacia el ápice $0,35 \mathrm{~mm}$ de diámetro; estigma punctiforme. Frutos ovoides con el cáliz relictual, de 4,7-4,9 × 3,3-3,5 mm. Semillas oblongas u ovoides, $0,60-0,81 \times 0,24-0,4 \mathrm{~mm}$; testa levemente reticulada, color crema.

Etimología. El epíteto específico es en honor a Brigitte Batiste, directora del Instituto de investigaciones de Recursos Biológicos Alexander von Humboldt, en agradecimiento al apoyo a la realización de inventarios de biodiversidad en el marco del proyecto Colombia BIO.

Distribución y hábitat. A lo largo de la Cordillera Occidental, flanco occidental y centro-norte de la Cordillera Central, flanco oriental, entre los 1330 y 2020 m de elevación, en zonas de Bosque Subandino y Andino. Recolectada siempre en zonas de bosques (Figura 3).

Fenología. Recolectada con flores en enero, julio, septiembre, octubre y diciembre. Recolectada con frutos en enero, abril, julio, septiembre, octubre, noviembre y diciembre.

Estado de conservación. Las localidades de procedencia corresponden a zonas de bosque Subandino y Andino, los cuales han sido intervenidos fuertemente. También es una especie que requiere buenas condiciones de conservación del bosque. Según lineamientos de la IUCN (2014), cumple los criterios B1b(iii), por lo que se categoriza en "Vulnerable" (VU). 

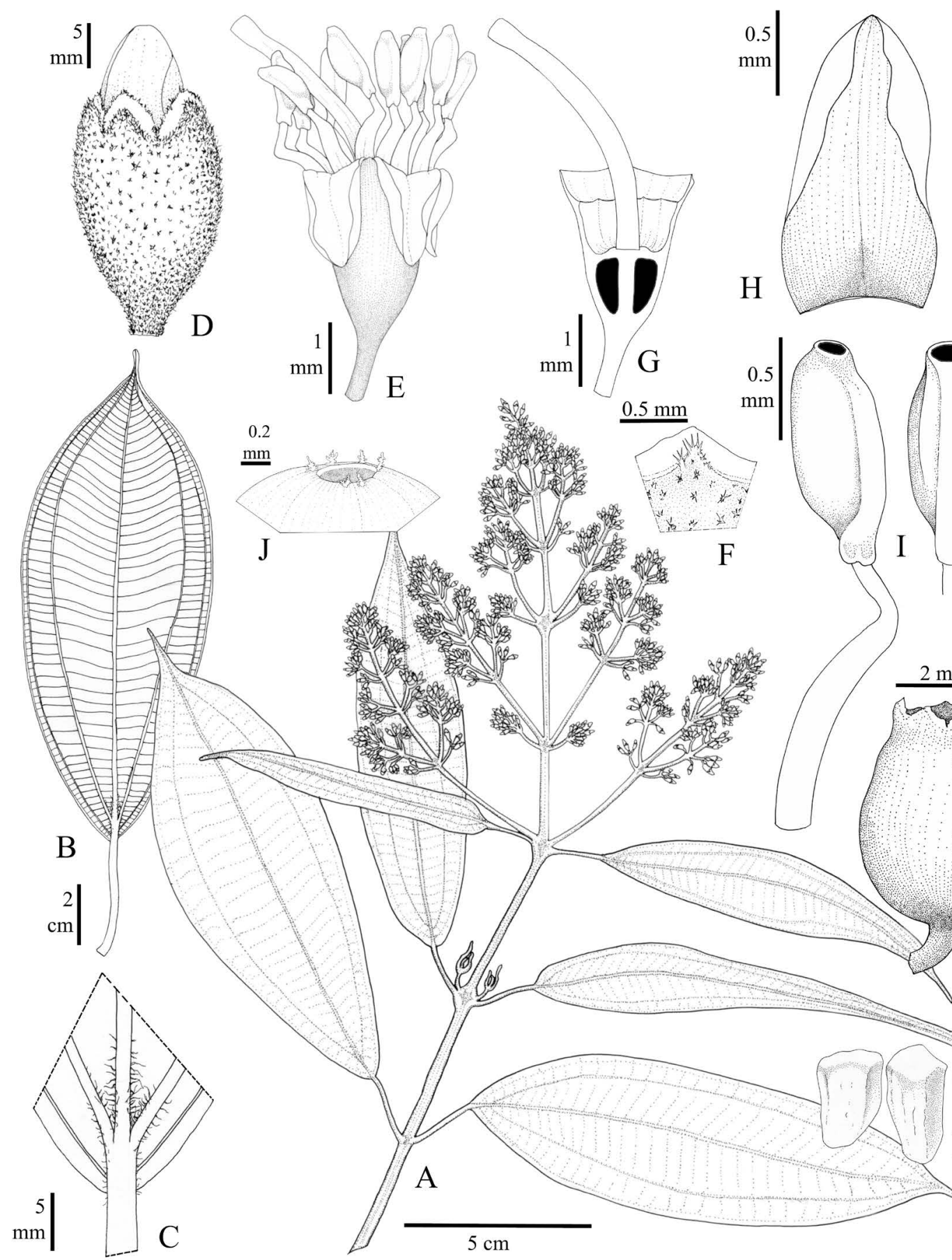

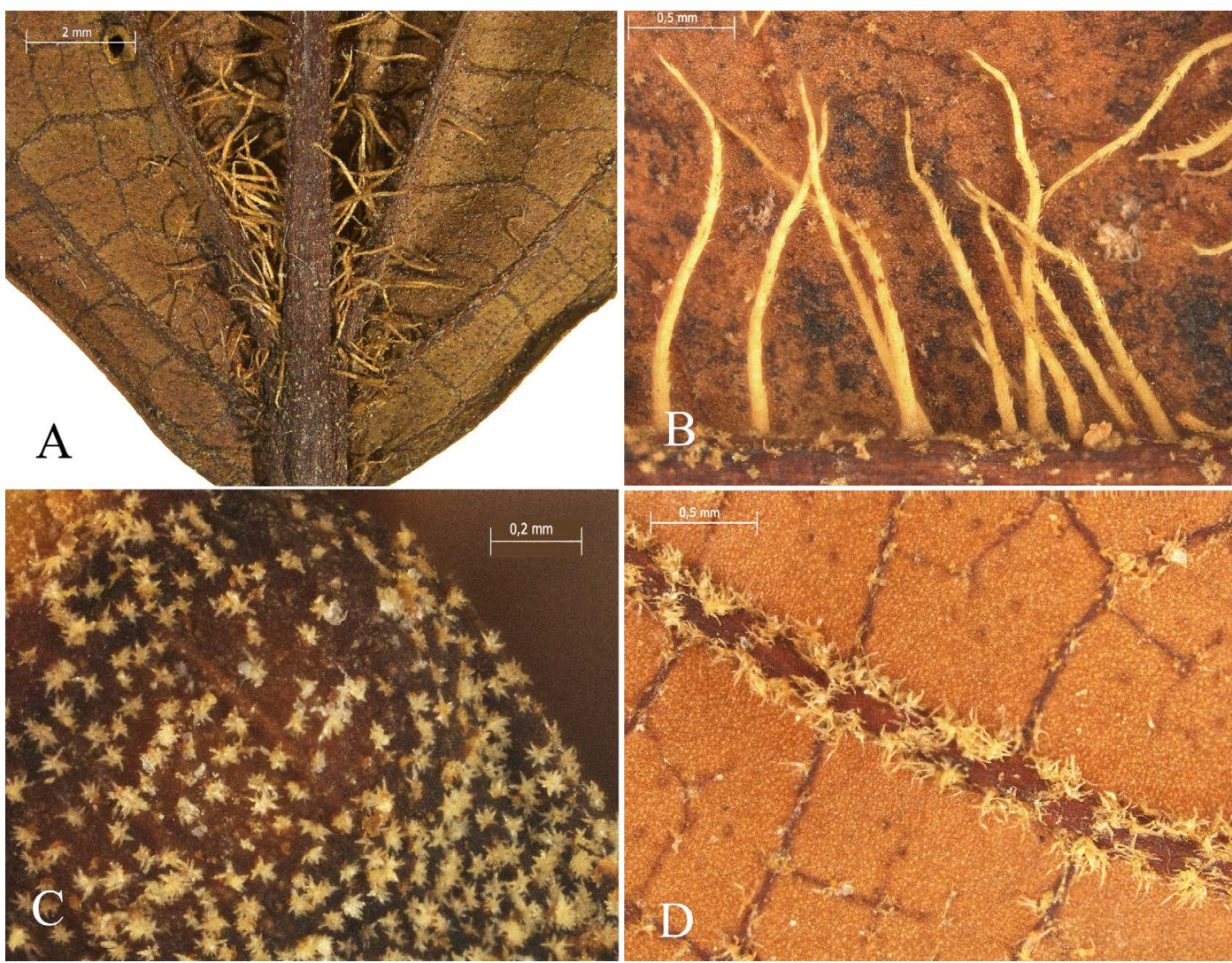

Figura 2. Tricomas en Miconia brigittei. A, Domacio pubescente del envés foliar; B. Tricomas del domacio del envés foliar; C. Tricomas del hipanto; D. Tricomas del envés foliar. Fotos basadas en: A - M. Velayos 7035 (FMB), B, C - H. Mendoza 19554 (FMB); D - H. Mendoza 15333 (FMB).

8642 (FMB!); 1800-1900 m, 9-IV-2005, fr, M. Cabrera 69 (FMB!); Reserva Natural La Planada, 1900 m, 22-IX-1995, fr, H. Mendoza 1437 (FMB!); 30-VII-1995, fl, H. Mendoza 882 (FMB!). Reserva Natural La Planada, sendero natural, $1^{\circ} 9^{\prime} 37^{\prime}$ 'N, 7759'13'W, 1850 m, 28-I-1997, fl, J. Bittner 2751 (FMB!). Valle del Cauca. La Cumbre, Corregimiento de Bitaco, vereda Chicoral, 3’33'56’N, 76³5'3'W, 2020 m, 23-VII-2003, st, H. Mendoza 15333 (FMB).

\section{Discusión}

Esta especie se reconoce por el secado rojizo de las hojas en los ejemplares de herbario, nervadura 5-plinervia, domacios pubescentes (tuff domatia) en la base de las venas secundarias por el envés, pétalos reflexos, conectivo minutamente prolongado en la base doral, y ovario 3-locular. Por la forma de los estambres cortos y oblongos con un pequeño poro apical, el cáliz lobulado y el tipo de inflorescencia, esta especie se asocia con Miconia sección Amblyarrhena (Naudin) Triana ex Hook. $f$ siguiendo el sistema de Cogniaux (1891). Miconia sección Amblyarrhena se encuentra ampliamente diversificado en los Andes, es un grupo parafilético de especies (Goldenberg et al. 2008) y no se conocen buenas descripciones de muchas de sus especies, aspectos que no permiten, con certeza, establecer las especies más cercanas filogenéticamente a este nuevo taxón.
Entre las especies de Miconia de Colombia con hojas 5-plinervias, las más parecidas vegetativamente son $M$. tenuis Triana y $M$. centrodesmoides Wurdack, las cuales carecen de domacios pubescentes por el envés y el secado de los ejemplares no es rojizo.

Vegetativamente también es semejante a especies de hojas 3-5-plinervias de Ecuador y Perú como son $M$. barbeyana Cogn. (sin domacios pubescente, los pétalos son granulosos y los estambre levemente dimorfos), $M$. cajanumana Wurdack (con domacios pubescentes pero con indumento de ramas e inflorescencia distinto, ápice de las hojas no acuminado, flores sésiles y más grandes, filamentos glandular pubérulos y anteras subuladas), $M$. conformis Wurdack (sin domacios, estambres dimorfos y anteras filiformes), M. laxa Wurdack (no tiene domacios pubescentes, las hojas son más pequeñas y no secan oscuro, hipanto glabro, pétalos suborbiculares), M. lívida Triana (sin domacios, hojas corto pecioladas, inflorescencias pauciflora, y flores mucho más grades con largos estigmas), $M$. ombrophila Wurdack (no tiene domacios pubescente, el estigma es expandido, y el estilo y estambres tienen tricomas glandulares), y M. pausana Wurdack (sin domacios, indumento muy diferente, pétalos redondeados, estigma expandido). M. cookii Gleason y M. terera Naudin del Perú, 


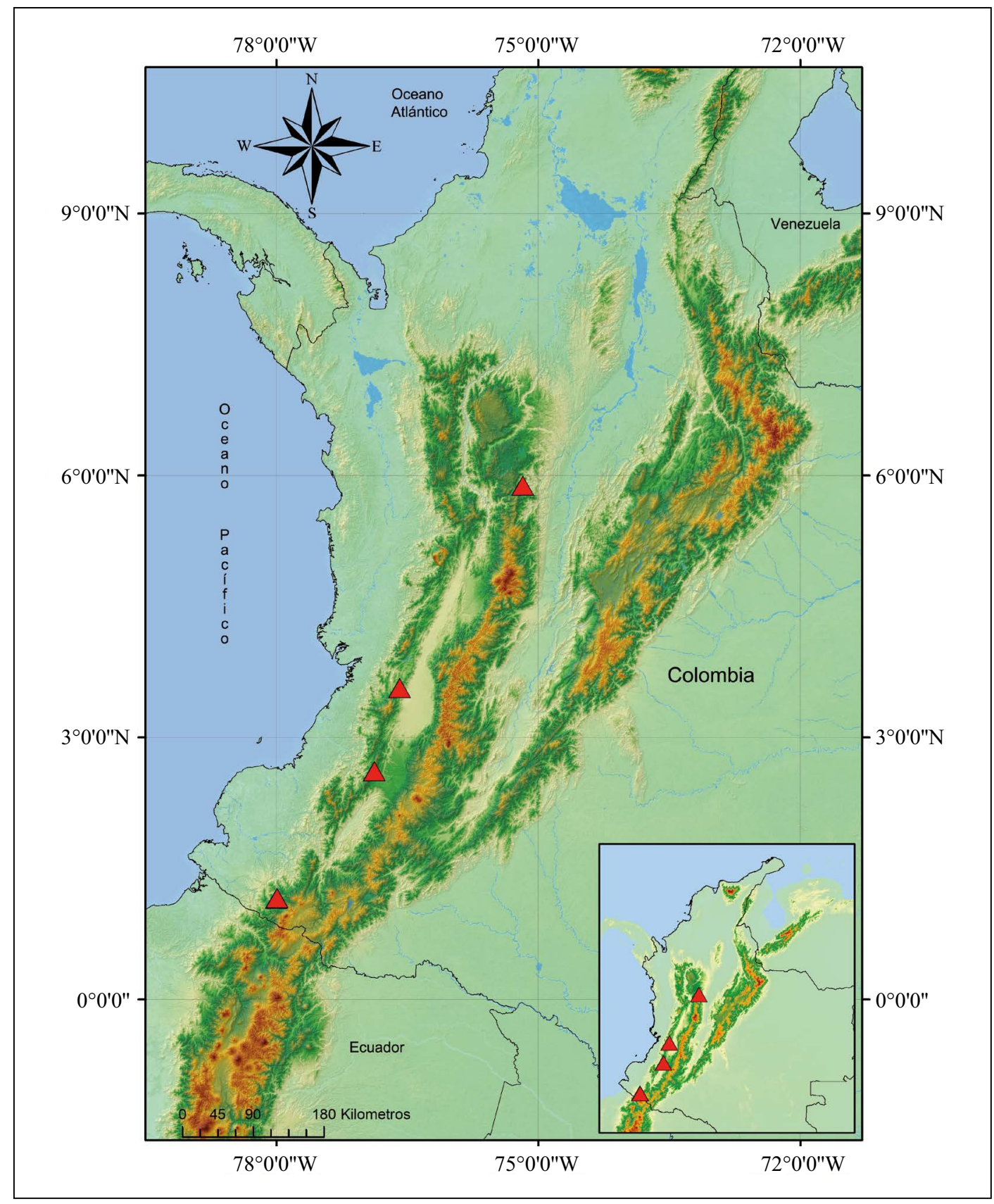

Figura 3. Distribución geográfica de Miconia brigittei (triángulos rojos).

también son parecidas vegetativamente, pero difieren por la ausencia de domacios pubescentes en el envés foliar y por ser plantas completamente glabras.

\section{Agradecimientos}

Expresamos sinceros agradecimientos al Instituto Alexander von Humboldt y especialmente a los coordinadores del proyecto Colombia BIO: Hernando García, Carlos Lasso, Mailyn González y Javier Barrigas. Extendemos nuestros agradecimientos a la comunidad de la vereda El Porvenir en Antioquia quien nos apoyó durante la expedición de Colombia
BIO. A Andrés Rymel Acosta por la elaboración de los mapas de distribución. A COLCIENCIAS, financiadora del Convenio Especial Colombia-BIO COLCIENCIAS-IAVH FP44842-109-2016 (IAVH 16-062) y a Fabián Michelangeli por sus sugerencias durante la revisión de este manuscrito.

\section{Contribución de los autores}

Humberto Mendoza-Cifuentes, compilación de información, colecciones, escritura general del artículo. Mauricio Posada, información general, discusión y escritura general del artículo. 


\section{Conflicto de intereses}

Los autores declaran no tener conflicto de intereses.

\section{Referencias}

Almeda, F. (2009). Melastomataceae. In G. Davidse, M. Sousa S, S. Knapp, F. Chiang [eds.], Flora mesoamericana, vol. 4 (1): 164-338. Universidad Nacional Autónoma de México, México City.

Almeda, F., Alvear, M., Mendoza-Cifuentes, H., Penneys, D. S., Michelangeli, F. (2016). Melastomataceae. En: R. Bernal, S. R. Gradstein, M. Celis (Eds.). Catálogo de plantas y líquenes de Colombia (pp. 1585-1664). Bogotá. Instituto de Ciencias Naturales, Universidad Nacional de Colombia.

Cogniaux, A. (1891). Melastomataceae. Pages 1-1256 in A de Candolle, C de Candolle, eds. Monographie Phanerogamarum. Vol 7. Masson, Paris.

Goldenberg, R., Penneys, D. S., Almeda, F., Judd, W. S., Michelangeli, F. A. (2008). Phylogeny of Miconia (Melastomataceae): patterns of stamen diversification in a megadiverse neotropical genus. International Journal of Plant Sciences, 169: 963-979.
Goldenberg, R., Almeda, F., Caddah, M. K., Martins, A. B., Meirelles, J., Michelangeli, F. A., Weiss, M. (2013). Nomenclator botanicus for the neotropical genus Miconia (Melastomataceae: Miconieae). Phytotaxa, 106: 1-171.

Michelangeli, F. A., Almeda, F., Alvear, M., Bécquer, E. R., Burke, J., Caddah, M. K., Goldenberg, R., Ionta, G. M., Judd, W. S., Majure, L. C., Meirelles, J., Nicolas A. N., Ocampo, G., Penneys, D. S, Dan Skean Jr., J., Ulloa Ulloa, C. (2016). (2462) Proposal to conserve Miconia, nom. cons. against the additional names Maieta and Tococa (Melastomataceae: Miconieae). TAXON, 65 (4): 892-893.

IUCN. (2012). Iucn red list categories and criteria: Version 3.1. Second edition. IUCN, Gland, Switzerland and Cambridge, UK.

IUCN Standards and Petitions Subcommittee. (2014). Guidelines for using the IUCN red list categories and criteria. Version 11. Prepared by the standards and petitions subcommittee. Downloadable from http://www.Iucnredlist.Org/documents/ redlistguidelines.Pdf. IUCNCogniux (1891)

Wurdack, J. J. (1986). Atlas of hairs for Neotropical Melastomataceae. Smithsonian Contributions to Botany, 63: 1-80. 